https://doi.org/10.32541/recie.2017.v1i1.pp18-33

\title{
EL PAPEL DEL DIRECTOR ESCOLAR EN LOS APRENDIZAJES DE LOS ESTUDIANTES
}

\section{The role of the school principal in academic achievement}

- Daniel Morales*

Instituto Dominicano de Evaluación

e Investigación de la Calidad Educativa

República Dominicana

Correo-e: daniel.morales@ideice.gob.do

- Claudia Curiel

Instituto Dominicano de Evaluación

e Investigación de la Calidad Educativa

República Dominicana

Correo-e: claudia.curiel@ideice.gob.do
- Samuel Bonilla

Universidad Pontificia

Católica Madre y Maestra

República Dominicana

Correo-e: SamuelBonilla@pucmm.edu.do

\section{Resumen}

Con el fin de aprovechar la aplicación de la Evaluación Diagnóstica de Segundo Año de Educación Media en mayo del 2013, se administró un instrumento que pregunta a los directores acerca de su percepción sobre la efectividad, al realizar diversas tareas de gestión en su escuela. En adición, se consideraron algunas características de los directores, como el género, si obtuvo el cargo por concurso, si ha realizado algún programa en gestión educativa, años de experiencia y su última titulación académica. Tomando en cuenta también diversas características de los estudiantes, se encontró que las titulaciones académicas de maestría y doctorado del director están relacionadas con mejores resultados de aprendizaje, y que los directores que se consideran más efectivos en tareas de gestión pedagógica están relacionados con menores resultados de aprendizaje de los estudiantes.

Palabras clave: eficacia del liderazgo; cualidades de liderazgo; liderazgo escolar; liderazgo instruccional.

\section{Abstract}

In May 2013, during the application of the Second Year Diagnostic Assessment of Secondary Education, we gave an assessing instrument to school directors to measure their belief of effectiveness in performing various management tasks in their school. In addition, the instrument considered characteristics such as gender, academic degree, years of experience; if school directors obtained the position by contest, and if they have been trained in educational management. Also considering diverse characteristics of students, we found that school director's masters or doctoral academic degrees are related to better results of learning, and directors that considered themselves as more effective in performing tasks of pedagogical management are related to lower results of student learning.

Keywords: Leadership effectiveness; leadership qualities; school leadership; instructional leadership.

* Autor de correspondencia.

ISSN (impreso): 2636-2139

ISSN (en línea): 2636-2147

Recibido: 10/05/2017

Sitio web: https://revistas.isfodosu.edu.do/recie

Aprobado: 16/08/2017

\section{COMO CITAR}

Morales, D., Curiel, C., \& Bonilla, S. (2017). El papel del director escolar en los aprendizajes de los estudiantes. Revista Caribeña de Investigación Educativa (RECIE), 1(1), 18-33. https://doi.org/10.32541/recie.2017. v111.pp18-33 


\section{Introducción}

Con el fin de aprovechar la logística de aplicación de la Evaluación Diagnóstica de Educación Media (2013), el Centro de Investigación en Liderazgo y Gestión Educativa (CILGE) introdujo en los protocolos de la Evaluación Diagnóstica un instrumento orientado a la medición de sus percepciones sobre la efectividad del trabajo del director, en sus diversas labores de gestión en la escuela. Con estos datos y otros que reúnen características del director se trató de identificar la relación que tiene cada característica en los aprendizajes de los estudiantes.

El principal antecedente de esta investigación es el trabajo de Morales, Martí, Bernal, Español y Curiel (2014), en el que se aplicó el inventario sobre percepciones de los directores escolares a la segunda y tercera cohorte de la Escuela de Directores del Instituto Superior de Formación Docente Salomé Ureña (ISFODOSU). Con esos datos y otras características de los directores y de las escuelas, se había encontrado que los directores que se perciben más efectivos realizando tareas de gestión organizacional están relacionados con un mayor puntaje en las pruebas nacionales. Sin embargo, en Morales et al. (2014) se advertía sobre las limitaciones de la investigación. En primer lugar, la muestra de directores no fue representativa a nivel nacional, y además había un sesgo en la selección de directores, por lo que no podían generalizarse los resultados del estudio. En segundo lugar, los datos de pruebas nacionales usados eran promedios agregados a nivel escolar, ya que no fue posible contar con microdatos relativos a los estudiantes ni con características correspondientes a ellos, lo que se presume generó estimaciones sesgadas e inconsistentes. En tercer lugar, dado que las pruebas nacionales se realizan con fines de promoción y no de comparación, se sospecha que cualquier resultado que logre obtenerse siempre permanecerá en duda. Afortunadamente, la Evaluación Diagnóstica realizada a estudiantes de segundo año de Educación Media permite hacer frente a cada uno de estos problemas y avanzar algunos pasos en la comprensión del papel que juega el director escolar dominicano en el logro aprendizajes de los estudiantes.

Este trabajo está circunscrito a la literatura especializada de liderazgo y gestión educativa, y su relación con resultados en la escuela. En particular está relacionado con dos investigaciones. La primera es la de Grissom, Kalogrides y Loeb (2014), que presenta un marco conceptual para identificar la contribución del director en los resultados de pruebas estandarizadas, que miden niveles de aprendizaje de los estudiantes. El enfoque específico de esta literatura utiliza los resultados de dichas pruebas para medir el desempeño de los gestores escolares. Según estos autores, es difícil identificar en qué medida el desempeño de una escuela podría ser atribuido directamente a la acción del director o a factores que escapan de su control. Por ejemplo, ¿son responsables los directores de la efectividad de maestros que ellos no contratan directamente? En la segunda investigación (Grissom \& Loeb, 2011), se describen las diferencias entre diversas autopercepciones sobre la efectividad de los directores al realizar tareas de gestión organizacional, administrativa, pedagógica, de relaciones internas y externas, con algunas características como sexo, años de experiencia e instrucción académica. También se realiza un análisis de regresión para estudiar la relación entre cada una de las tareas de gestión y los resultados de los estudiantes. Si bien consiguen que las tareas más relacionadas con el éxito escolar tienen más que ver con las tareas de gestión organizacional, sus aportes y hallazgos pertenecen a un contexto diferente al dominicano.

En este trabajo se presentan estadísticas de diversas características de los directores, las escuelas y los estudiantes, así como resultados en base a pruebas de diferencia de medias en la proporción de respuestas correctas que tienen los estudiantes en Matemáticas, Lengua Espańola, Ciencias Naturales 
y Ciencias Sociales como una medida que aproxima el nivel de aprendizaje logrado, que traten de identificar factores determinantes del desempeño educativo. Más aun, se realizaron análisis de regresión tomando en cuenta características de los estudiantes, de los directores y sus percepciones sobre la efectividad al realizar diversas tareas de gestión en la escuela para tratar de develar la relación que podría existir entre cada característica y los resultados educativos.

En las pruebas de diferencia de medias no se logró identificar, de manera consistente con teorías y supuestos, ninguna característica del director relacionada con mejores o peores resultados en el promedio de respuestas correctas para cada una de las áreas de la evaluación diagnóstica. Sin embargo, del análisis de regresión emergen dos resultados interesantes. El primero es que, luego de tomar en cuenta características socio-demográficas de los estudiantes, las escuelas que tienen directores que poseen un grado de instrucción académico más alto (maestría y/o doctorado), sus estudiantes obtienen en promedio $2 \%$ más respuestas correctas que los estudiantes de las escuelas donde sus directores tienen un grado de instrucción menor. No se consiguió ninguna relación estadística entre la proporción de respuestas correctas y las siguientes características del director: género, si obtuvo la posición por concurso, si tiene formación en gestión educativa, años de experiencia y si asistió a la Escuela de Directores. Como segundo resultado se presentan regresiones tomando en cuenta la autopercepción de los directores sobre su efectividad al realizar diversas tareas de gestión. Se consiguió que en las escuelas donde los directores se consideran más efectivos realizando tareas de gestión pedagógica, los estudiantes obtienen, en términos estadísticos, una proporción menor de respuestas correctas en la Evaluación Diagnóstica. Aunque estos resultados son consistentes con lo encontrado por Grissom, Grissom et al. (2013), no logran conseguir la misma relación estadística encontrada por Grissom y Loeb (2011) y Morales et al. (2014) sobre la importancia relativa entre las tareas de gestión organizacional y los resultados en los aprendizajes.

Los resultados encontrados en esta investigación tienen dos tipos de potencialidad: sobre investigaciones futuras que mejoren y refinen las preguntas en los instrumentos, de manera que puedan obtenerse mejores datos para producir información más precisa; y sobre implicaciones de políticas de RR. HH. del MINERD para lograr directores escolares con los perfiles y características más apropiados para el logro de los objetivos del sistema educativo.

\section{Revisión de la literatura}

En Grissom y Loeb (2011) se reseñan los paradigmas desde los cuales se concibe qué deberían hacer los directores en la escuela, o a cuáles áreas o tipos de tareas deberían dedicar más tiempo, atención y esfuerzo para ser más efectivos en el logro de los objetivos de la escuela. La determinación empírica de estos factores tiene implicaciones de política respecto a la formación y preparación de directores de escuelas, y para las políticas de RR. HH. de selección de directores escolares. Hallinger y Murphy (1985) sostienen que el paradigma que más soporte empírico ha tenido, y que llama la atención de académicos y hacedores de políticas educativas, establece que el trabajo más importante de los directores escolares, y al que deben dedicarse más, son las tareas relacionadas con el logro de los aprendizajes en el salón de clase. En esta misma línea, Marks y Printy (2003) sostienen que lo que el director debe hacer durante el día es una gestión pedagógica para apoyar el logro de los estudiantes y la habilidad de los profesores de enseñar. También, Robinson, Lloyd y Rowe (2008) concluyen que, a medida que los líderes centren más sus relaciones, su trabajo y su aprendizaje en 
la actividad principal o core business de la escuela, es decir, la enseñanza y el aprendizaje, mayor será su influencia en los resultados de los estudiantes.

Grissom y Loeb (2011) desafían esta visión con un enfoque complementario. Para ellos, las actividades principales del trabajo del día a día de un director no tienen que ver con la "enseñanza y el aprendizaje", sino con la gestión de la burocracia, es decir, hacer que la escuela funcione: manejar finanzas, estar pendientes de los asuntos del personal, mantener la planta física, cumplir con el papeleo y las regulaciones exigidas por el Ministerio de Educación, atender a los padres y tutores, supervisar la enseñanza, integrar la comunidad, etc. En este sentido, Grissom y Leob (2011) observan que la mayor parte del trabajo del director, en realidad, no se consume en actividades y tareas relacionadas con la actividad principal de la escuela. Como señala March (1978), las escuelas son burocracias, y, por tanto, necesitan gerentes que las manejen. Así, la mayor parte de tiempo de un director se consume en hacer que el componente administrativo funcione; por lo que las tareas relacionadas con la enseńanza y el aprendizaje son unas entre tantas.

Grissom y Loeb (2011) consiguen, sistemáticamente, que las tareas relacionadas con la gerencia organizacional estén positiva y estadísticamente relacionadas con una diversidad de indicadores de éxito escolar en las escuelas de Miami-Dade Country. Grissom et al. (2013) encuentran que el tiempo de dedicación a la gestión pedagógica no predice aprendizajes. Específicamente, concluyen en que el tiempo empleado en supervisar al profesor en el aula tiene un efecto negativo en los aprendizajes, mientras que el coaching y la evaluación a los docentes están relacionados positivamente con los aprendizajes en los estudiantes.

Las implicaciones del trabajo de Grissom y Loeb (2011), Grissom et al (2013), y la presente investigación, son pertinentes para revisar la formación de directores escolares en República Dominicana e identificar características relevantes que contribuyan al establecimiento de los perfiles de los candidatos que tengan la potencialidad de ser más efectivos en lograr calidad educativa.

\section{Método}

La Evaluación Diagnóstica del Primer Ciclo de Educación Media fue realizada en mayo del año 2013. La prueba diagnóstica se aplicó a más de nueve mil estudiantes de segundo año de educación media, en una muestra de escuelas representativas a nivel nacional, estratificada por sector público y privado. El objetivo general fue realizar una evaluación diagnóstica acerca del aprendizaje de los estudiantes y su relación con otras variables socioeconómicas y educativas al concluir el Primer Ciclo de la Educación Media, que permitieran orientar y tomar decisiones de mejora de la calidad educativa. Los directores respondieron a dos encuestas: una que levanta información sobre características generales de la escuela, datos socio-demográficos del director, percepciones sobre diversos asuntos de la institución a su cargo; y otra, sobre percepciones acerca de su propia efectividad al gestionar la escuela. En la tabla 1 se presentan los datos que lograron recopilarse de los directores de las mismas.

De esta muestra de directores, el 43\% afirmó haber obtenido el cargo por concurso, el 77\% haber realizado un programa de formación en gestión educativa, el 17\% haber participado en la Escuela de Directores del ISFODOSU. También, se recogió información sobre los años de experiencia como director, y específicamente del centro en cuestión, así como del grado de la última titulación académica. El 34\% de los directores de la muestra posee una maestría y/o doctorado. El 17\% de las escuelas incluidas en la muestra son del sector privado, y el $80 \%$ están ubicadas en zonas urbanas. 
Tabla 1

Características de los directores de la muestra

\begin{tabular}{|c|c|c|c|c|c|c|}
\hline VARIABLE & PROPORCIÓN & $\begin{array}{l}\text { DESVIACIÓN } \\
\text { ESTANDAR }\end{array}$ & MIN & MAX & OBS & $\begin{array}{l}\text { DATOS } \\
\text { PERDIDOS }\end{array}$ \\
\hline Sexo director: Mujeres & $49.13 \%$ & 0.501 & 0 & 1 & 173 & 10 \\
\hline Concurso de Oposición & $43.02 \%$ & 0.497 & 0 & 1 & 172 & 11 \\
\hline Formación de Gestión Educativa & $76.69 \%$ & 0.424 & 0 & 1 & 163 & 20 \\
\hline Escuela de Directores & $17.06 \%$ & 0.377 & 0 & 1 & 170 & 13 \\
\hline Años de Experiencia como Director & 10.735 & 8.940 & 1 & 38 & 151 & 32 \\
\hline $\begin{array}{l}\text { Años de Experiencia como Director } \\
\text { en el Centro }\end{array}$ & 8.570 & 7.406 & 1 & 38 & 151 & 32 \\
\hline Muy satisfechos & $57.23 \%$ & 0.496 & 0 & 1 & 173 & 10 \\
\hline Maestro Normal & $0.00 \%$ & 0.000 & 0 & 0 & 182 & 1 \\
\hline Certificado Docente & $1.10 \%$ & 0.105 & 0 & 1 & 182 & 1 \\
\hline Habilitación Docente & $0.55 \%$ & 0.074 & 0 & 1 & 182 & 1 \\
\hline Lincenciatura & $35.22 \%$ & 0.480 & 0 & 1 & 182 & 1 \\
\hline Especialidad & $24.18 \%$ & 0.429 & 0 & 1 & 182 & 1 \\
\hline Maestría & $33.52 \%$ & 0.473 & 0 & 1 & 182 & 1 \\
\hline Doctorado & $0.55 \%$ & 0.074 & 0 & 1 & 182 & 1 \\
\hline Especialidad, maestría y doctorado & $58.24 \%$ & 0.495 & 0 & 1 & 182 & 1 \\
\hline Maestría y Doctorado & $34.07 \%$ & 0.475 & 0 & 1 & 182 & 1 \\
\hline Centros Privados & $16.67 \%$ & 0.374 & 0 & 1 & 182 & 9 \\
\hline $\begin{array}{l}\text { Colegios } \backslash \text { Escuelas } \\
\text { Urbanas }\end{array}$ & $80.75 \%$ & 0.396 & 0 & 1 & 182 & 22 \\
\hline
\end{tabular}

Fuente: elaboración propia.

Asimismo, a los estudiantes se les aplicó una encuesta que levanta información socioeconómica (ver tabla 2). 


\section{Tabla 2}

Características de los estudiantes de la muestra

\begin{tabular}{|c|c|c|c|c|c|c|}
\hline VARIABLE & $\begin{array}{l}\text { Promedio/ } \\
\text { Proporcion }\end{array}$ & $\begin{array}{l}\text { DESVIACIÓN } \\
\text { ESTANDAR }\end{array}$ & MIN & MAX & OBS & $\begin{array}{l}\text { DATOS } \\
\text { PERDIDOS }\end{array}$ \\
\hline Sexo Estudiantes: Mujeres & $54.98 \%$ & 0.498 & 0 & 1 & 9068 & 135 \\
\hline Edad Estudiantes & 16.32 & 1.722 & 14 & 20 & 8976 & 227 \\
\hline$\%$ de estudiantes con hijos & $8.67 \%$ & 0.281 & 0 & 1 & 8308 & 895 \\
\hline $\begin{array}{l}\% \text { que viven con el padre en su } \\
\text { hogar }\end{array}$ & $48.50 \%$ & 0.500 & 0 & 1 & 9203 & 0 \\
\hline Internet en el hogar & $61.16 \%$ & 0.487 & 0 & 1 & 7109 & 2094 \\
\hline $\begin{array}{l}\% \text { de estudiantes en Centros } \\
\text { Privados }\end{array}$ & $9.86 \%$ & 0.298 & 0 & 1 & 8783 & 420 \\
\hline Sexo Estudiantes: Mujeres & $81.77 \%$ & 0.386 & 0 & 1 & 8081 & 1122 \\
\hline
\end{tabular}

Fuente: elaboración propia.

La edad promedio de los estudiantes de segundo año de Educación Media es 16.32 años, el 9\% de los estudiantes tienen hijos, el 48.5\% declara que su padre vive en el hogar y el 61\% tiene internet en su casa. Este es el conjunto de variables socioeconómicas que considera este estudio.

De forma particular, el propósito principal de la evaluación diagnóstica es medir el rendimiento de los estudiantes en el área de Matemáticas, Lengua Española, Ciencias de la Naturaleza y Ciencias Sociales. En la tabla 3 se presentan los promedios de la proporción de respuestas correctas por área.

Tabla 3

Proporción de respuestas correctas de los estudiantes de la muestra

\begin{tabular}{|c|c|c|c|c|c|c|}
\hline VARIABLE & $\begin{array}{l}\text { Promedio/ } \\
\text { Proporción }\end{array}$ & $\begin{array}{l}\text { DESVIACIÓN } \\
\text { ESTANDAR }\end{array}$ & MIN & MAX & OBS & $\begin{array}{l}\text { DATOS } \\
\text { PERDIDOS }\end{array}$ \\
\hline $\begin{array}{l}\% \text { de respuestas correctas en } \\
\text { Matemáticas }\end{array}$ & $26.11 \%$ & 0.071 & 0 & 0.68 & 9117 & 86 \\
\hline $\begin{array}{l}\% \text { de respuestas correctas en } \\
\text { Lengua Espańola }\end{array}$ & $41.42 \%$ & 0.160 & 0 & 0.92 & 9117 & 86 \\
\hline $\begin{array}{l}\% \text { de respuestas correctas en } \\
\text { Ciencias Naturales }\end{array}$ & $32.66 \%$ & 0.104 & 0 & 0.76 & 9117 & 86 \\
\hline $\begin{array}{l}\% \text { de respuestas correctas en } \\
\text { Ciencias Sociales }\end{array}$ & $40.24 \%$ & 0.128 & 0 & 0.88 & 9117 & 86 \\
\hline $\begin{array}{l}\text { Promedio \% de respuestas } \\
\text { correctas }\end{array}$ & $35.11 \%$ & 0.084 & 0.03 & 0.705 & 9117 & 86 \\
\hline
\end{tabular}

Fuente: elaboración propia.

Según el Diseño Teórico-Metodológico de la Evaluación Diagnóstica del Primer Ciclo de Educación Media (2013), el conjunto y subconjuntos de ítems para cada una de las áreas evalúan el dominio de los estudiantes sobre los conocimientos que se espera que los estudiantes tengan, 
dado el currículo aprobado por el MINERD. En este sentido, el área que más logra cumplir con los objetivos del currículo es Lengua Española, y el que menos lo logra es el área de Matemáticas.

En la tabla 4 se presentan diferencias de media en la proporción de respuestas correctas para un grupo de características de los directores.

\section{Tabla 4}

Diferencias de media en logros educativos caracterizados por directores

\begin{tabular}{|c|c|c|c|c|c|c|}
\hline VARIABLE & & MATEMÁTICAS & $\begin{array}{l}\text { LENGUA } \\
\text { ESPAÑOLA }\end{array}$ & NATURALES & SOCIALES & PROMEDIO \\
\hline Sexo director & $\begin{array}{l}\text { Hombres } \\
\text { Mujeres }\end{array}$ & $\begin{array}{l}0.260 \\
0.262\end{array}$ & $\begin{array}{l}0.417 \\
0.409\end{array}$ & $\begin{array}{l}0.326 \\
0.327\end{array}$ & $\begin{array}{l}0.401 \\
0.404\end{array}$ & $\begin{array}{l}0.351 \\
0.351\end{array}$ \\
\hline Concurso de Oposición & $\begin{array}{l}\text { No } \\
\text { Si }\end{array}$ & $\begin{array}{l}0.264 \\
0.257\end{array}$ & $\begin{array}{l}0.422 \\
0.403\end{array}$ & $\begin{array}{l}0.332 \\
0.320\end{array}$ & $\begin{array}{l}0.408 \\
0.396\end{array}$ & $\begin{array}{l}0.357 \\
0.344\end{array}$ \\
\hline $\begin{array}{c}\text { Formación en Gestión } \\
\text { Educativa }\end{array}$ & $\begin{array}{l}\text { No } \\
\text { Si }\end{array}$ & $\begin{array}{l}0.261 \\
0.260\end{array}$ & $\begin{array}{l}0.416 \\
0.412\end{array}$ & $\begin{array}{l}0.328 \\
0.327\end{array}$ & $\begin{array}{l}0.401 \\
0.403\end{array}$ & $\begin{array}{l}0.351 \\
0.351\end{array}$ \\
\hline Escuela de Directores & $\begin{array}{c}\text { No } \\
\text { Si }\end{array}$ & $\begin{array}{l}0.260 \\
0.266\end{array}$ & $\begin{array}{l}0.411 \\
0.426\end{array}$ & $\begin{array}{l}0.324 \\
0.336\end{array}$ & $\begin{array}{l}0.399 \\
0.419\end{array}$ & $\begin{array}{l}0.349 \\
0.362\end{array}$ \\
\hline $\begin{array}{l}\text { Especialidad, Maestría y } \\
\text { Doctorado. }\end{array}$ & $\begin{array}{l}\mathrm{No} \\
\mathrm{Si}\end{array}$ & $\begin{array}{l}0.257 \\
0.264\end{array}$ & $\begin{array}{l}0.399 \\
0.424\end{array}$ & $\begin{array}{l}0.316 \\
0.333\end{array}$ & $\begin{array}{l}0.387 \\
0.412\end{array}$ & $\begin{array}{l}0.340 \\
0.358\end{array}$ \\
\hline Maestría y Doctorado. & $\begin{array}{l}\text { No } \\
\text { Si }\end{array}$ & $\begin{array}{l}0.257 \\
0.269\end{array}$ & $\begin{array}{l}0.400 \\
0.442\end{array}$ & $\begin{array}{l}0.320 \\
0.338\end{array}$ & $\begin{array}{l}0.390 \\
0.426\end{array}$ & $\begin{array}{l}0.342 \\
0.369\end{array}$ \\
\hline Sector & $\begin{array}{l}\text { Público } \\
\text { Privado }\end{array}$ & $\begin{array}{l}0.258 \\
0.290\end{array}$ & $\begin{array}{l}0.405 \\
0.483\end{array}$ & $\begin{array}{l}0.323 \\
0.359\end{array}$ & $\begin{array}{l}0.397 \\
0.446\end{array}$ & $\begin{array}{l}0.346 \\
0.394\end{array}$ \\
\hline Zona & $\begin{array}{l}\text { Rural } \\
\text { Urbana }\end{array}$ & $\begin{array}{l}0.261 \\
0.261\end{array}$ & $\begin{array}{l}0.403 \\
0.415\end{array}$ & $\begin{array}{c}0.326 \\
0.26\end{array}$ & $\begin{array}{l}0.307 \\
0.403\end{array}$ & $\begin{array}{l}0.347 \\
0.351\end{array}$ \\
\hline
\end{tabular}

Fuente: elaboración propia.

La proporción de respuestas correctas para todas las áreas de la Evaluación Diagnóstica de Educación Media es significativamente mayor en los estudiantes de las escuelas con directores que no obtuvieron su puesto por concurso de oposición (incluye a directores de escuelas privadas que no obtienen su nombramiento por concurso); la proporción de respuestas correctas es significativamente mayor en las escuelas en las que los directores declaran haber asistido a la Escuela de Directores. Sin embargo, no parece plausible que se puedan atribuir esas mejores calificaciones a la Escuela de Directores ya que tanto la prueba como la Escuela de Directores empezaron el mismo ańo y con pocos meses de diferencia. Lo que podría explicar esa diferencia es, más bien, un sesgo de selección en los participantes en la Escuela de Directores, en la que probablemente se seleccinó a los directores de las mejores escuelas.

Las diferencias que más llaman la atención son las que se encuentran en los grupos de directores que poseen un grado de instrucción académica mayor, lo que podría indicar que la formación académica del director es un dato determinante en los aprendizajes del estudiante.

Las restricciones a las que se enfrenta un director de una escuela pública podrían estar relacionadas con su capacidad de gestión, lo que podría a su vez impactar los aprendizajes de los estudiantes.

La proporción de respuestas correctas en las escuelas del sector privado es significativamente mayor que en las escuelas del sector público. Ahora bien, no se puede afirmar que esta diferencia pueda ser atribuible sólo a la capacidad de gestión de los directores en ambos sectores; pudiera ser un indicador relacionado con características socioeconómicas de los estudiantes. 
A los directores incluidos en la muestra se les aplicó el inventario usado por Morales et al. (2014). El inventario pregunta: "¿Qué tan efectivo piensa que es usted en cumplir correctamente cada una de las siguientes tareas (...)? Las respuestas posibles por tarea son "Muy efectivo", "Efectivo", "Algo efectivo", "Poco efectivo". A cada una de estas respuestas se les asignan los valores de 4, 3, 2, 1 respectivamente. Con estos valores se realizan promedios por tarea, y se obtienen los componentes principales.

\section{Tabla 5}

Promedio de componentes principales de las tareas de gestión

\begin{tabular}{|c|c|c|c|c|c|c|}
\hline VARIABLE & $\begin{array}{l}\text { Promedio/ } \\
\text { Proporción }\end{array}$ & $\begin{array}{l}\text { DESVIACIÓN } \\
\text { ESTÁNDAR }\end{array}$ & MIN & MAX & OBS & $\begin{array}{l}\text { DATOS } \\
\text { PERDIDOS }\end{array}$ \\
\hline $\begin{array}{l}\text { Componente Principal } \\
\text { Organización }\end{array}$ & 0.031 & 1.685 & -4.707 & 2.314 & 154 & 29 \\
\hline $\begin{array}{l}\text { Componente Principal } \\
\text { Administración }\end{array}$ & 0.003 & 1.735 & -6.080 & 1.793 & 164 & 19 \\
\hline $\begin{array}{l}\text { Componente Principal Intrucción } \\
\text { Academica }\end{array}$ & -0.012 & 2.102 & -5.829 & 3.399 & 160 & 23 \\
\hline $\begin{array}{l}\text { Componente Principal Relaciones } \\
\text { Internas }\end{array}$ & 0.033 & 1.682 & -6.242 & 2.025 & 164 & 19 \\
\hline $\begin{array}{l}\text { Componente Principal Relaciones } \\
\text { Externas }\end{array}$ & 0.034 & 1.506 & -3.847 & 2.193 & 166 & 17 \\
\hline
\end{tabular}

Fuente: elaboración propia.

A continuación, son presentadas las pruebas de diferencia de medias de los componentes principales de cada una de las áreas de gestión para diversas características del director. 
Tabla 6

Diferencia de medias en los componentes principales de autopercepción sobre la efectividad

\begin{tabular}{|c|c|c|c|c|c|c|}
\hline VARIABLE & & $\begin{array}{l}\text { COMPONENTE } \\
\text { PRINCIPAL } \\
\text { ORGANIZACIÓN }\end{array}$ & $\begin{array}{r}\text { COMPONENTE } \\
\text { PRINCIPAL } \\
\text { ADMINISTRACIÓN }\end{array}$ & $\begin{array}{r}\text { COMPONENTE } \\
\text { PRINCIPAL } \\
\text { INSTRUCCIÓN } \\
\text { ACADÉMICA }\end{array}$ & $\begin{array}{l}\text { COMPONEN- } \\
\text { TE PRINCIPAL } \\
\text { RELACIONES } \\
\text { INTERNAS }\end{array}$ & $\begin{array}{r}\text { COMPONENTE } \\
\text { PRINCIPAL } \\
\text { RELACIONES } \\
\text { EXTERNAS }\end{array}$ \\
\hline Sector & $\begin{array}{l}\text { Público } \\
\text { Privado }\end{array}$ & $\begin{array}{c}0.021 \\
-0.0244\end{array}$ & $\begin{array}{c}-0.0993 \\
0.4051\end{array}$ & $\begin{array}{c}-0.1445 \\
0.4406\end{array}$ & $\begin{array}{c}-0.0645 \\
0.3407\end{array}$ & $\begin{array}{c}0.0692 \\
-0.2944\end{array}$ \\
\hline Zona & $\begin{array}{c}\text { Rural } \\
\text { Urbana }\end{array}$ & $\begin{array}{l}0.2348 \\
0.0195\end{array}$ & $\begin{array}{c}-0.048 \\
-0.00343\end{array}$ & $\begin{array}{r}-0.105 \\
-0.00495\end{array}$ & $\begin{array}{c}-0.0404 \\
0.105\end{array}$ & $\begin{array}{c}0.2591 \\
-0.0142\end{array}$ \\
\hline Sexo Director & $\begin{array}{l}\text { No } \\
\text { Sí }\end{array}$ & $\begin{array}{c}0.0591 \\
-0.00467\end{array}$ & $\begin{array}{l}-0.2123 \\
0.2347\end{array}$ & $\begin{array}{l}0.4032 \\
0.3809\end{array}$ & $\begin{array}{c}-0.1369 \\
0.1877\end{array}$ & $\begin{array}{r}0.2591 \\
-01983\end{array}$ \\
\hline $\begin{array}{l}\text { Concurso de } \\
\text { Oposición }\end{array}$ & $\begin{array}{l}\text { No } \\
\text { Sí }\end{array}$ & $\begin{array}{l}0.0938 \\
0.1421\end{array}$ & $\begin{array}{l}0.1826^{*} \\
-0.2821\end{array}$ & $\begin{array}{l}0.003335 \\
-0.1005\end{array}$ & $\begin{array}{l}0.0506 \\
0.0325\end{array}$ & $\begin{array}{c}-01392 \\
0.267\end{array}$ \\
\hline $\begin{array}{l}\text { Escuela de Direc- } \\
\text { tores }\end{array}$ & $\begin{array}{l}\text { No } \\
\text { Sí }\end{array}$ & $\begin{array}{r}-0.3686 \\
0.1086\end{array}$ & $\begin{array}{l}-0.4166 \\
0.0788\end{array}$ & $\begin{array}{l}-0.5073 \\
0.0728\end{array}$ & $\begin{array}{l}-01654 \\
0.0743\end{array}$ & $\begin{array}{c}-0.3344 \\
0.1078\end{array}$ \\
\hline $\begin{array}{l}\text { Formación en } \\
\text { Gestión Educa- } \\
\text { tiva }\end{array}$ & $\begin{array}{l}\text { No } \\
\text { Sí }\end{array}$ & $\begin{array}{r}0.0859 \\
-0.2621\end{array}$ & $\begin{array}{l}0.0422 \\
-02664\end{array}$ & $\begin{array}{l}0.0832 \\
0.4439\end{array}$ & $\begin{array}{c}0.0736 \\
-0.0798\end{array}$ & $\begin{array}{c}0.0692 \\
-0.1821\end{array}$ \\
\hline $\begin{array}{l}\text { Especialidad, } \\
\text { Maestría y Doc- } \\
\text { torado }\end{array}$ & $\begin{array}{l}\text { No } \\
\text { Sí }\end{array}$ & $\begin{array}{l}-0.1606 \\
0.1819\end{array}$ & $\begin{array}{r}-00892 \\
0.071\end{array}$ & $\begin{array}{l}-01751 \\
0.1024\end{array}$ & $\begin{array}{c}-0.1661 \\
0.1814\end{array}$ & $\begin{array}{l}0.0433 \\
0.0265\end{array}$ \\
\hline $\begin{array}{l}\text { Maestría y Doc- } \\
\text { torado }\end{array}$ & $\begin{array}{l}\text { No } \\
\text { Sí }\end{array}$ & $\begin{array}{c}-0.1101 \\
0.3068\end{array}$ & $\begin{array}{r}0.029 \\
-0.051\end{array}$ & $\begin{array}{c}-0.1703 \\
0.2987\end{array}$ & $\begin{array}{c}-0.16 \\
0.4157\end{array}$ & $\begin{array}{l}0.0341 \\
0.0325\end{array}$ \\
\hline
\end{tabular}

Fuente: elaboración propia.

La prueba de diferencia de medias, no logra identificar diferencias significativas para ninguna área de gestión en diversas características de los directores.

\subsection{Mínimos cuadrados ordinarios (MCO)}

La sección anterior trató de mostrar e identificar características de los directores relacionadas con los resultados educativos, y determinar fuentes de diferencias entre las áreas de gestión en que los directores se perciben más efectivos. Ambas aproximaciones son un insumo importante para el establecimiento de controles estadísticos que deben tomarse en cuenta a la hora de tratar de explicar el desempeño de los estudiantes y su relación con el trabajo del director.

Siguiendo a Morales et al. (2014) se realiza un análisis de regresión con el método de mínimos cuadrados ordinarios $(\mathrm{MCO})$.

Sea $Y_{i j}$ la proporción de respuestas correctas en la Evaluación Diagnóstica para el estudiante $i$ de la escuela bajo la gestión del director del $j$, y sea $T$ la tarea o el subconjunto de tareas (colapsadas en el componente principal), se estima la relación entre las tareas y los resultados escolares por medio del siguiente modelo:

$$
Y_{i j}=\alpha+\beta T+\delta X_{j}+\omega S_{i j}+\varepsilon
$$

Donde $X_{i}$ es un vector de características del director y/o la escuela. El parámetro de interés a estimar es $\hat{\beta}_{M C O}$, y $S_{i j}$ es un conjunto de características de los estudiantes. 
En realidad, $\beta T$ es igual a $\sum_{j=1 ; z=1}^{j=n ; z=5} \beta_{z} T_{j z}$, donde $\beta_{z}$ 's son los coeficientes asociados a los componentes principales de los conjuntos de tareas (tareas organizacionales, $\beta_{1}$; tareas de administración, $\beta_{2}$; tareas de instrucción académica, $\beta_{3}$; tareas de relaciones internas, $\beta_{4}$; $\mathrm{y}$ tareas de relaciones externas, $\beta_{5}$ ); y donde ${ }^{T_{j z}}$ son los componentes principales (tareas organizacionales del director de la escuela $j, T_{j_{1}}$; de tareas de administración de la escuela $j,{ }_{j_{2}}$; de tareas de instrucción académica de la escuela $j, T_{j 3}$; de tareas de relaciones internas de la escuela $j,{ }_{j} T_{4}$ y de tareas de relaciones externas de la escuela $j, T_{j_{5}}$.

Cuando se toman en cuenta los factores relevantes que hacen diferentes a las escuelas y a los estudiantes, los coeficientes $\hat{\beta}_{M C O}$ podrían llegar a ser plausiblemente un estimador insesgado y consistente del impacto de la efectividad de las tareas del director en los resultados escolares, teniendo presente que se tiene una medida de la autopercepción como aproximación de efectividad de gestión.

\subsubsection{Análisis de estimaciones MCO}

En la tabla 7 se muestran las estimaciones MCO de 11 especificaciones. La primera sólo toma en cuenta las siguientes características de los estudiantes: edad, si tienen hijos, si su padre vive en el hogar, si tiene internet en su casa y si está en una escuela privada. En conjunto, los coeficientes estimados son estadísticamente significativos al $1 \%$ y el r-cuadrado es 0.1058 , lo que indica que un $10.58 \%$ de la variabilidad observada en el promedio de la proporción de respuestas correctas en todas las pruebas está explicada por estas características de los estudiantes.

El coeficiente estimado asociado a una escuela privada resultó estadísticamente significativo al $1 \%$, lo que sugiere que, en promedio, un estudiante de una escuela privada obtiene $4 \%$ más de respuestas correctas que uno de una escuela pública. Esta variable se considera como una característica del estudiante, en el entendido de que es una aproximación de una característica socioeconómica de la familia. Tener internet en el hogar también es una seńal económica de la familia, y aunque la relación entre este factor y la proporción de respuestas correctas es estadísticamente significativa (5\%), la magnitud del coeficiente es marginal. En cambio, la edad del estudiante puede constituir una diferencia relativamente importante en el logro de los conocimientos. Cada año de sobreedad está relacionado negativamente con $1.3 \%$ menos de respuestas correctas. 
Tabla 7

Mínimos Cuadrados Ordinarios (Variable dependiente: Promedio de Áreas)

\begin{tabular}{|c|c|c|c|c|c|c|c|c|c|c|}
\hline & 1 & 2 & 3 & 4 & 5 & 6 & 7 & 8 & 9 & 10 \\
\hline p-valor F & $(0.00)^{* * *}$ & $(0.00)^{* * *}$ & $(0.00)^{* * *}$ & $(0.00)^{* * *}$ & $(0.00)^{* * *}$ & $(0.00)^{* * *}$ & $(0.00)^{* * *}$ & $(0.00)^{* * *}$ & $(0.00)^{* * *}$ & $(0.00)^{* * *}$ \\
\hline r-cuadrado & 0.1058 & 0.1059 & 0.1054 & 0.1061 & 0.1125 & 0.1172 & 0.1068 & 0.1159 & 0.1288 & 0.1379 \\
\hline $\begin{array}{l}\mathrm{N} \text {. } \\
\text { Observaciones }\end{array}$ & 6022 & 6022 & 5795 & 5582 & 5297 & 5357 & 5672 & 6022 & 4693 & 4693 \\
\hline $\begin{array}{l}\text { N. de Clusters } \\
\text { (Escuelas) }\end{array}$ & 173 & 173 & 165 & 161 & 149 & 149 & 164 & 173 & 131 & 131 \\
\hline \multicolumn{11}{|l|}{ VARIABLES } \\
\hline $\begin{array}{l}\text { Edad } \\
\text { Estudiante }\end{array}$ & $\begin{array}{l}-0.013^{* * *} \\
(0.00)\end{array}$ & $\begin{array}{l}-0.014^{* * *} \\
(0.00)\end{array}$ & $\begin{array}{l}-0.014 \\
(0.00)\end{array}$ & $\begin{array}{l}-0.013^{* * *} \\
(0.00)\end{array}$ & $\begin{array}{l}-0.013^{* * *} \\
(0.00)\end{array}$ & $\begin{array}{l}-0.014 \\
(0.00)\end{array}$ & $\begin{array}{l}-0.013 \\
(0.00)\end{array}$ & $\begin{array}{l}-0.013 \\
(0.00)\end{array}$ & $\begin{array}{l}-0.01^{* * *} \\
(0.00)\end{array}$ & $\begin{array}{r}-0.013 \\
(0.00)\end{array}$ \\
\hline $\begin{array}{l}\text { Hijos } \\
\text { Estudiantes }\end{array}$ & $\begin{array}{l}0.000 \\
(0.94)\end{array}$ & $\begin{array}{l}-0.001 \\
(0.90)\end{array}$ & $\begin{array}{l}-0.001 \\
(0.78)\end{array}$ & $\begin{array}{l}-0.001 \\
(0.78)\end{array}$ & $\begin{array}{l}-0.001 \\
(0.84)\end{array}$ & $\begin{array}{l}-0.000 \\
(0.97)\end{array}$ & $\begin{array}{l}-0.002 \\
(0.63)\end{array}$ & $\begin{array}{l}0.000 \\
(0.96)\end{array}$ & $\begin{array}{l}0.00 \\
(0.57)\end{array}$ & $\begin{array}{l}-0.002 \\
(0.78)\end{array}$ \\
\hline $\begin{array}{l}\text { Papás estu- } \\
\text { diantes en el } \\
\text { hogar }\end{array}$ & $\begin{array}{l}0.001 \\
(0.55)\end{array}$ & $\begin{array}{l}-0.001 \\
(0.56)\end{array}$ & $\begin{array}{l}0.001 \\
(0.77)\end{array}$ & $\begin{array}{l}0.001 \\
(0.61)\end{array}$ & $\begin{array}{l}-0.001 \\
(0.63)\end{array}$ & $\begin{array}{l}0.001 \\
(0.72)\end{array}$ & $\begin{array}{l}0.001 \\
(0.56)\end{array}$ & $\begin{array}{l}0.002 \\
(0.49)\end{array}$ & $\begin{array}{l}0.00 \\
(0.91)\end{array}$ & $\begin{array}{l}-0.000 \\
(0.96)\end{array}$ \\
\hline $\begin{array}{l}\text { Internet en el } \\
\text { Hogar }\end{array}$ & $\begin{array}{l}0.007^{* *} \\
(0.02)\end{array}$ & $\begin{array}{l}0.007^{* *} \\
(0.02)\end{array}$ & $\begin{array}{l}0.007^{* *} \\
(0.02)\end{array}$ & $\begin{array}{l}0.008^{* *} \\
(0.01)\end{array}$ & $\begin{array}{l}0.008^{* *} \\
(0.01)\end{array}$ & $\begin{array}{l}0.008 \\
(0.01)\end{array}$ & $\begin{array}{l}0.007 \\
(0.03)\end{array}$ & $\begin{array}{l}0.006^{* *} \\
(0.04)\end{array}$ & $\begin{array}{l}0.01^{* *} \\
(0.01)\end{array}$ & $\begin{array}{c}0.007^{* *} \\
(0.04)\end{array}$ \\
\hline $\begin{array}{l}\text { Centro } \\
\text { Privado }\end{array}$ & $\begin{array}{l}0.04^{* * *} \\
(0.00)\end{array}$ & $\begin{array}{l}0.04^{* * *} \\
(0.00)\end{array}$ & $\begin{array}{l}0.04^{* *} \\
(0.00)\end{array}$ & $\begin{array}{l}0.04^{* * *} \\
(0.00)\end{array}$ & $\begin{array}{l}0.05^{* *} \\
(0.00)\end{array}$ & $\begin{array}{l}0.05 \\
(0.00)\end{array}$ & $\begin{array}{l}0.04^{* * *} \\
(0.00)\end{array}$ & $\begin{array}{l}0.04^{* * *} \\
(0.00)\end{array}$ & $\begin{array}{l}0.04^{* * *} \\
(0.00)\end{array}$ & $\begin{array}{l}0.04^{* * *} \\
(0.00)\end{array}$ \\
\hline \multicolumn{11}{|c|}{ CARACTERÍSTICAS DEL DIRECTOR } \\
\hline $\begin{array}{l}\text { Sector Direc- } \\
\text { tor: } \\
\text { Mujeres }\end{array}$ & $\begin{array}{l}0.00 \\
(0.74)\end{array}$ & & & & & & & & $\begin{array}{l}0.00 \\
(0.83)\end{array}$ & $\begin{array}{l}0.00 \\
(0.67)\end{array}$ \\
\hline $\begin{array}{l}\text { Concurso de } \\
\text { Oposición }\end{array}$ & & $\begin{array}{l}0.00 \\
(0.84)\end{array}$ & & & & & & & $\begin{array}{l}-0.01 \\
(0.36)\end{array}$ & $\begin{array}{l}-0.01 \\
(0.21)\end{array}$ \\
\hline $\begin{array}{l}\text { Formación } \\
\text { En Gestión } \\
\text { Educativa }\end{array}$ & & & $\begin{array}{l}0.00 \\
(0.72)\end{array}$ & & & & & & $\begin{array}{l}0.00 \\
(0.52)\end{array}$ & $\begin{array}{l}0.00 \\
(0.50)\end{array}$ \\
\hline $\begin{array}{l}\text { Años de Expe- } \\
\text { riencia como } \\
\text { Director }\end{array}$ & & & & $\begin{array}{l}0.00 \\
(0.35)\end{array}$ & & & & & & \\
\hline $\begin{array}{l}\text { Años de Expe- } \\
\text { riencia como } \\
\text { Director en el } \\
\text { Centro }\end{array}$ & & & & & $\begin{array}{l}0.00 \\
(0.96)\end{array}$ & & & & $\begin{array}{l}0.00 \\
(0.91)\end{array}$ & $\begin{array}{l}0.00 \\
(0.58)\end{array}$ \\
\hline $\begin{array}{l}\text { Escuela de } \\
\text { Directores }\end{array}$ & & & & & & $\begin{array}{l}0.02^{*} \\
(0.07)\end{array}$ & & & $\begin{array}{l}0.01 \\
(0.18)\end{array}$ & $\begin{array}{l}0.01 \\
0.17\end{array}$ \\
\hline $\begin{array}{l}\text { Espacialidad, } \\
\text { Maestria y } \\
\text { Doctorado }\end{array}$ & & & & & & & $\begin{array}{l}0.02^{* * *} \\
(0.00)\end{array}$ & & $\begin{array}{l}0.01 \\
(0.01)\end{array}$ & \\
\hline $\begin{array}{l}\text { Maestria y } \\
\text { Doctorado }\end{array}$ & & & & & & & & $\begin{array}{l}0.02^{* * *} \\
(0.00)\end{array}$ & & $\begin{array}{l}0.02^{* * *} \\
(0.00)\end{array}$ \\
\hline
\end{tabular}

Fuente: elaboración propia.

P-valores entre paréntesis. Se presentan los coeficientes estimados asociados a cada variable. Se señalan con asteriscos aquellas coeficientes que sean estadisticamente significativos: ***(\%1). 
De la segunda a la novena regresión se presentan estimaciones sobre la contribución, que pudieran tener diversas características de los directores en la proporción de respuestas correctas. Solo emerge una relación estadística fuerte cuando se toma en cuenta el último grado académico del director. La regresión ocho presenta el coeficiente estimado asociado al grupo de directores que declaran que su última titulación es especialidad, maestría o doctorado. La proporción de respuestas correctas de los estudiantes, en el centro que es gestionado por un director con estos grados académicos, es $2 \%$ más de respuestas correctas que los estudiantes que están en centros cuyo director tiene un grado académico menor. Lo mismo ocurre en la regresión nueve, cuando solo se considera el grupo de directores con maestría y doctorado en relación con los demás.

Las regresiones 10 y 11 exploran si las asociaciones estadísticas encontradas para el grado académico más alto del director se mantienen cuando se toman en cuenta todas las características del director consideradas. La regresión 12 muestra que la proporción de respuestas correctas es 1\% mayor en las escuelas donde el grado académico más alto es maestría y doctorado, que en el grupo que incluye a estos y a los que tienen especialidad (regresión 10), lo que parece indicar que el grado académico del director es un factor importante en logro de los aprendizajes de los estudiantes.

La tabla 8 recoge el rol que podría jugar la autopercepción sobre la efectividad del director en ejecutar diversas tareas de gestión en la escuela.

\section{Tabla 8}

Mínimos Cuadrados Ordinarios (Variable Dependiente: Promedio)

\begin{tabular}{|c|c|c|c|c|}
\hline & 1 & 2 & 3 & 4 \\
\hline p-valor F & $(0.07)^{*}$ & $(0.00)^{* * *}$ & $(0.00)^{* * *}$ & $(0.00)^{* * *}$ \\
\hline r-cuadrado & 0.0186 & 0.10973 & 0.1081 & 0.117 \\
\hline $\begin{array}{l}\mathrm{N} \text {. } \\
\text { Observaciones }\end{array}$ & 6893 & 5017 & 5017 & 5017 \\
\hline N. de Clusters (Escuelas) & 130 & 126 & 126 & 126 \\
\hline \multicolumn{5}{|l|}{ VARIABLES } \\
\hline Organización & $\begin{array}{c}-0.013^{* * * *} \\
(0.00)\end{array}$ & $\begin{array}{c}-0.014^{* * * *} \\
(0.00)\end{array}$ & $\begin{array}{l}-0.014 \\
(0.00)\end{array}$ & $\begin{array}{c}-0.013^{* * * *} \\
(0.00)\end{array}$ \\
\hline Administración & $\begin{array}{l}0.000 \\
(0.94)\end{array}$ & $\begin{array}{c}-0.001 \\
(0.90)\end{array}$ & $\begin{array}{l}-0.001 \\
(0.78)\end{array}$ & $\begin{array}{l}-0.001 \\
(0.78)\end{array}$ \\
\hline $\begin{array}{l}\text { Institución } \\
\text { Académica }\end{array}$ & $\begin{array}{l}0.001 \\
(0.55)\end{array}$ & $\begin{array}{c}-0.001 \\
(0.56)\end{array}$ & $\begin{array}{l}0.001 \\
(0.77)\end{array}$ & $\begin{array}{l}0.001 \\
(0.61)\end{array}$ \\
\hline $\begin{array}{l}\text { Relaciones } \\
\text { Internas }\end{array}$ & $\begin{array}{c}0.007 * * \\
(0.02)\end{array}$ & $\begin{array}{c}0.007 * * \\
(0.02)\end{array}$ & $\begin{array}{c}0.007 * * \\
(0.02)\end{array}$ & $\begin{array}{c}0.008^{* *} \\
(0.01)\end{array}$ \\
\hline $\begin{array}{l}\text { Relaciones } \\
\text { Externas }\end{array}$ & $\begin{array}{c}0.04 * * * \\
(0.00)\end{array}$ & $\begin{array}{c}0.04 * * * \\
(0.00)\end{array}$ & $\begin{array}{c}0.04^{* *} \\
(0.00)\end{array}$ & $\begin{array}{c}0.04^{* * *} \\
(0.00)\end{array}$ \\
\hline
\end{tabular}

Fuente: elaboración propia.

P-Valores entre paréntesis. Se presentan los coeficientes estimados asociados a cada variable. Se señalan con asteriscos aquellos coeficientes que sean estadísticamente significativos: ***(1\%),**(5\%),*(10\%) respectivamente. Los errores estándares con los que se realizan la pruebas de significación individual de cada coeficiente se calculan por medio de cluster a nivel escolar. La regresión 1 no controla por ninguna otra característica, la 2 controla por edad de estudiante, si tiene internet en el hogar, si el centro educativo es privado; la 3 usa los mismos controles de la 2 más si el director realiza alguna especialización, maestría o doctorado; y la 4 usa los mismos controles de la 3, pero se sustituye el último control por maestrías y doctorado solamente. 
Estas estimaciones muestran que en las escuelas donde los directores se autoperciben más efectivos en gestionar asuntos pedagógicos, la proporción de respuestas correctas es menor. Estos resultados son consistentes con lo encontrado por Grissom, Loeb y Master (2013), pero diferentes a los encontrados por Grissom y Loeb (2011) y Morales et al. (2014).

\section{Análisis y recomendaciones}

Al centrar el análisis en el papel que juega el director y sus características en el logro de los aprendizajes de los estudiantes, es importante destacar que los resultados de este estudio no son conclusivos y deben servir de base para futuras investigaciones relacionadas.

En los últimos años, la política educativa se ha hecho más transparente. Consecuentemente, los procesos de selección de personal también; por lo que se han mejorado los procesos de selección. En principio, se esperaría que un concurso de oposición permita tener candidatos con las competencias necesarias para producir mejores resultados en la escuela. En lo que se refiere a la proporción de respuestas correctas, no parece haber diferencias entre las escuelas donde el director obtuvo el cargo por concurso y aquellas en las que se produjo un nombramiento directo. Habría que explorar el año que obtuvo el cargo por concurso, y si hubo diferencias en los criterios de selección para los diversos procesos de concurso.

Este resultado tiene una implicación de política de RR. HH., y posibles ideas de investigación a futuro. Si bien, RR. HH. del MINERD está mejorando los procesos de selección, tanto de directores como de docentes, no se conoce si los criterios de selección usados son efectivos para el logro de resultados escolares. Futuras investigaciones podrían relacionar los nuevos criterios de selección de personal del MINERD con los resultados educativos a nivel escolar.

La formación en gestión educativa no parece jugar un papel importante en el logro de los resultados de aprendizajes de la escuela. Lamentablemente, la encuesta no pregunta si la formación en gestión fue recibida antes o después del nombramiento como director, si fue un requisito para obtener el cargo ni la cantidad de formación en gestión, ni siquiera el grado de instrucción. RR. HH. podría hacer un esfuerzo para recopilar datos actualizados de los directores de escuelas y documentar con evidencias qué tipo (cantidad, fecha de obtención, grado académico, universidad) de formación en gestión tienen los directores de escuelas de República Dominicana. De esta manera se podrá establecer si esta formación tiene o no incidencia en los resultados escolares en interacción con otras características de los directores.

Tanto los ańos de ejercicio como de experiencia como director del centro educativo parecen no guardar relación con los aprendizajes de los estudiantes. Sin embargo, Morales (2014) presenta pruebas de diferencia de medias que muestran que en las escuelas donde los directores son más jóvenes, los estudiantes obtienen 1\% más de respuestas correctas que en las escuelas donde los directores son mayores. Estos resultados no son conclusivos, pero ayudan a orientar otras preguntas de investigación sobre la edad y la experiencia de los directores que podrían ser consideraciones de políticas de RR. HH. del MINERD. Por un lado, podría buscarse cómo la experiencia y la edad ayuda o no con los resultados de la escuela. Por otro, si la obtención del cargo de director implica su permanencia hasta la jubilación, quizá habría que revisar las políticas de

carrera directiva dentro del MINERD con la política docente, y verificar su flexibilidad para 
retornar al aula u otra posición dentro del sistema, si es que se comprueba que ya no es efectivo para gestionar la escuela. Esto tiene diversas implicaciones de supervisión, acompañamiento y evaluación de RR.HH.

En la muestra de directores utilizada para este estudio, el 17.06\% declaró haber participado en la Escuela de Directores. La prueba de diferencia de medias logra detectar que en las escuelas donde los directores participaron en este programa, los estudiantes obtienen significativamente $1.58 \%$ más de respuestas correctas que las de directores que no participaron en el programa; mientras que el coeficiente estimado asociado a la participación en la Escuela de Directores es estadísticamente significativo (10\%) en la regresión 7, y pierde peso cuando se toman en cuenta otras características.

Uno de los problemas asociados a esta estimación es que los directores que han participado en la Escuela de Directores no fueron seleccionados al azar. Por lo tanto, los resultados presentados en este trabajo no pueden tomarse como una evaluación de ese programa de formación. Más aun, tanto la evaluación diagnóstica como el programa fueron implementados en el mismo ańo, con lo cual no hay un rezago de tiempo apropiado para computar un cambio en los resultados que pueda ser atribuible a ese programa de formación. Pero aunque existiera el rezago, un sesgo de selección de participación de directores en el programa podría ser la explicación de cualquier diferencia que pueda aparecer. Por ejemplo, lo directores convocados podrían ser de las mejores escuelas, lo cual explicaría los mejores resultados, y no la incidencia de la Escuela de Directores.

La Escuela de Directores es una iniciativa importante del MINERD, cumple con un mandato del Plan Decenal 2008-2018 en relación a la formación de los directores de centros escolares. Es importante seguir monitoreando los resultados de esta iniciativa en la que hay un gran campo para la investigación experimental y para el diseño de formatos efectivos de enseñanza de habilidades de liderazgo y gestión de centro educativo. A la fecha, y al mejor entendimiento de los autores de este estudio, no existe ninguna investigación en República Dominicana que haya probado formatos efectivos en tal sentido.

La única característica del director que parece jugar un papel importante para explicar su relación con los aprendizajes es su última titulación académica. Ahora bien, no podría atribuirse a un título el logro de los resultados, ya que podrían estar operando otros factores que no se ven reflejados en una titulación. Por ejemplo, los directores con grados académicos más altos son los que más se esfuerzan, o los que están más motivados, o los que tienen más competencias directivas. Aquí existe un campo amplio de investigación que podría aportar a RR. HH. del MINERD otros factores característicos que los guíe en la elaboración de perfiles de directores efectivos. Habría que considerar otras dimensiones en interacción con el grado académico para determinar en qué medida, y a través de cuáles factores, la titulación del director impacta en los aprendizajes de los estudiantes.

En lo que se refiere a la gestión de los centros educativos, esta investigación solo consideró la incidencia de la autopercepción sobre la efectividad del director en la ejecución de diversas tareas en el marco de sus funciones. No se logró identificar ninguna relación estadística entre los directores que se consideran más o menos efectivos realizando tareas de gestión organizacional, administrativa, de relaciones internas y relaciones externas. En cambio, sí se estableció una relación negativa entre los directores que se consideran más efectivos en la realización de tareas de gestión pedagógica con los logros de los estudiantes. Estos resultados son desafiantes, pero consistentes con lo encontrado por Grissom et al. (2013). Ahora bien, la perspectiva de ellos es sobre el tiempo de dedicación a la gestión pedagógica. De manera particular ellos sostienen que 
el tiempo dedicado a gestionar la instrucción pedagógica no predice aprendizajes. Tampoco, el tiempo empleado en supervisar al profesor en el aula. En cambio, sí encuentran que el coaching y la evaluación a los docentes predicen aprendizajes en los estudiantes.

En contraste, este trabajo pregunta sobre la efectividad al realizar tareas, no sobre el tiempo que dedica a cada una. Investigaciones futuras podrían preguntar por el uso del tiempo en la gestión de diversas tareas de la gestión pedagógica, y tratar de entender por qué los directores que se perciben más efectivos en gestionar la instrucción pedagógica entregan peores resultados en los aprendizajes.

Las posibilidades de investigación en esta área son amplias, y podrían aportar información sobre cómo el trabajo del director, su asignación de tiempo, sus énfasis temáticos, guardan relación con los aprendizajes de los estudiantes.

Una recomendación general que se deriva de estos resultados y comentarios es que RR. HH. del MINERD desarrolle capacidades analíticas y de investigación que los lleve a aprovechar prospectivamente los datos contenidos en la Evaluación Diagnóstica y otras iniciativas de investigación local, así como lo que aporta la literatura especializada internacional. Hay una mina de información que puede ser aprovechada para obtener insumos para confirmar, reconducir o desechar diversas políticas de personal, que no sólo estén orientadas al logro de los resultados educativos, de manera que se puedan establecer relaciones de causalidad entre las políticas de RR. HH. del MINERD y los aprendizajes de los estudiantes.

Otra recomendación se refiera a los nuevos procesos de certificación y recertificación. Valdría la pena tratar de atar estos procesos a metodologías rigurosas de evaluación y seguimiento, y a una implementación ordenada que, además de permitir aprender del proceso, logre hacer rectificaciones oportunas que permitan el logro de los objetivos.

\section{Conclusiones}

La principal contribución de este trabajo fue presentar insumos para iniciar una discusión y una agenda de investigación sobre liderazgo y gestión educativa, con claras implicaciones de políticas de RR. HH. En este sentido, se trató de identificar relaciones entre características de los directores de centros educativos de República Dominicana y logros en los aprendizajes de los estudiantes, usando la Evaluación Diagnóstica de Educación Media (2013) a una muestra representativa a nivel nacional, estratificada por los sectores público y privado. Se encontró que la titulación académica del director está relacionada con mayores logros en los aprendizajes de los estudiantes. Sin embargo, en el análisis de los resultados se apuntó que la titulación por sí misma no explicaría la diferencia, ya que podría estar relacionada con mayores motivaciones o con otras cualidades del director. En lo que respecta a características de gestión de centros, en las escuelas en que los directores se perciben más efectivos ejecutando tareas de gestión pedagógica, sus estudiantes logran peores resultados en los aprendizajes.

Ninguno de estos hallazgos es conclusivo y están abiertos tanto a otros tratamientos analíticos, como a futuras iniciativas de investigación que traten de develar el papel del director en el logro de los estudiantes en República Dominicana. Lo que sí se puede concluir de forma cierta es que falta más iniciativa y evidencia para definir tanto el rol del director en los aprendizajes, como los mecanismos efectivos con que cuenta el MINERD para mejorar la efectividad del trabajo de los directores. 
Dado que hay mucho por aprender sobre el trabajo de los directores escolares en el contexto dominicano, el MINERD en general y RR. HH. en particular, deben demandar y/o desarrollar competencias analíticas que los lleve a buscar y usar evidencia empírica como la presentada en este trabajo.

\section{Referencias}

Grissom, J. A., \& Loeb, S. (2011). Triangulating principal effectiveness: How perspectives of parents, teachers, and assistant principals identify the central importance of managerial skills. American Educational Research Journal, 48(5), 1091-1123.

Grissom, J. A., Loeb, S., \& Master, B. (2013). Effective instructional time use for school leaders: Longitudinal evidence from observations of principals. Educational Researcher, 42(8), 433444.

Grissom, J. A., Kalogrides, D., \& Loeb, S. (2015). Using student test scores to measure principal performance. Educational Evaluation and Policy Analysis, 37(1), 3-28.

Hallinger, P., \& Murphy, J. (1985). Assessing the instructional management behavior of principals. The Elementary School Journal, 86(2), 217-247.

March, J. G. (1978). American public school administration: A short analysis. The School Review, $86(2), 217-250$.

Marks, H. M., \& Printy, S. M. (2003). Principal leadership and school performance: An integration of transformational and instructional leadership. Educational administration quarterly, 39(3), 370-397.

Ministerio de Educación de República Dominicana. (2013). Diseño Teórico-Metodológico. Evaluación Diagnóstica del Primer Ciclo de Educación Media. Dirección de Evaluación de la Calidad de la Educación. Santo Domingo: MINERD.

Morales, D. (2014) Potencialidades de Investigación con la Evaluación del Primer Ciclo de Educación Media. Review. Revista de Evaluación e Investigación Educativa, 1, 18-29.

Morales, D., Martí, C., Bernal, S., Español, A. y Curiel, C. (2014). Una aproximación preliminar a la efectividad de los directores educativos en la República Dominicana: implicaciones para una agenda de investigación y consideraciones de políticas. Revista de Gestión Educativa, (1), 2014.

Robinson, V. M., Lloyd, C. A., \& Rowe, K. J. (2008). The impact of leadership on student outcomes: An analysis of the differential effects of leadership types. Educational administration quarterly, 44(5), 635-674.

Secretaría de Estado de Educación. (2008). Plan Decenal de Educación 2008-2018. Un instrumento de trabajo en procura de la excelencia educativa. Santo Domingo: Secretaría de Estado de Educación de República Dominicana. 